\title{
Numerical investigation of passive stabilization of the surface temperature using PCM and metal foam
}

\author{
Mirosław Seredyński ${ }^{1, *}$ \\ ${ }^{1}$ Institute of Heat Engineering, Faculty of Power and Aeronautical Engineering, Warsaw University of Technology, \\ 21/25 Nowowiejska Str., 00-665 Warsaw, Poland
}

\begin{abstract}
Stabilization of the temperature of PVs is of great importance, due to strong relation between operating temperature and its efficiency. Due to low thermal conductivity of PCM, intensification of heat transfer to the operating material is needed. The four fully passive PV's temperature stabilization systems, based on the phase change material (PCM) are numerically investigated in this paper. Apart from the natural convection promotion, intensification of heat transfer is done with aluminium fins and aluminium foam. The simplified computational model based on the equilibrium formulation of energy transport equation, taking into account viscous and inertial fluid flow resistances in the porous material, is formulated and solved with the general purpose software - ANSYS Fluent. Proposed model is succesfully verified by comparing the results with available in literature numerical solutions.Simulations outcomes are presented, the temperature and liquid fraction distributions in proposed geometry configurations, temperature variations determined in selected points and averaged on the heated surface temperature plots. Presented results help to assign the best configuration.
\end{abstract}

\section{Introduction}

The temperature stabilization of electronic devices and photovoltaics is very important due to restricted operational requirements and considerable impact of temperature on their efficiency. As was reported by many researchers, e.g. Radziemska [1], the overall efficiency of a PV panel decreases by $0.5 \mathrm{pp}$ for every $1^{\circ} \mathrm{C}$ rise above $25^{\circ} \mathrm{C}$

The temperature stabilization methods can be roughly divided into active and passive ones. The latter is not dependent on the external power supply system, so it is highly recommended for systems which operating must be infallible and recovery of heat is not needed.

Among methods of passive cooling of PVs those based on phase change materials (PCM) increase their popularity. Basic properties of such materials is a melting temperature and latent heat. The phase change temperature must be tuned to temperature of upper source of heat and its difference should be as small as possible to minimize exergy losses and to assure high quality of temperature stabilization. The common materials considered for PV temperature stabilization are organic pcm's, which are chemically stable, non expensive, safe and does not cause corrosion in metals. Their main drawback is a low thermal conductivity, close to $0.2 \mathrm{~W} /(\mathrm{mK})$, so some effort is needed to intensify the heat transfer between the hot surface and the heat storage material [2].

In the extensive survey of cooling techniques for PVs presented by Sathe and Dhoble [3], those based on the pcm's were considered among the novel ones. Various solutions vere mentioned: based on natural convection promotion, utilization of fins and developed heat transfer area.

The passive temperature stabilization system for the building-integrated photovoltaics based on the pcm was analyzed experimentally and numerically by Huang et al. [4]. Three system configurations were studied: plain aluminium plate system, PV/PCM system without and with internal fins. Height of fins was lower than the depth of the container, so heat was not transferred immediately, through fins, to the rear wall. Authors presented validation of the numerical model for all considered cases with performed experiment, where the constant insolation $1000 \mathrm{~W} / \mathrm{m}^{2}$ was imposed on the vertical wall, and finally good conformity was reported. Analysis of the role of internal finning of the PCM container exposed to high thermal radiation flux on the temperature limiting was investigated by the same authors (Huang et al.) [5] and [6]. It was concluded, wider fins prevent from overheating for longer time [5] and lower distances between fins considerably reduce the front temperature [6].

Enhancement of heat transfer using metal foams (porosity 95\% and 10 PPI) saturated with PCMs, compared to pure PCMs, was investigated experimentally and numerically by Zhao et al. [7]. The sample was heated electrically from the bottom with a constant heat flux. Detailed analysis of charging and discharging of the composite showed the considerable increase in rate of the processes, 3-10 times for charging

\footnotetext{
Corresponding author: msered@itc.pw.edu.pl
} 
and 2 times for discharging. The numerical model of heat transfer in the composite based on the nonequilibrium energy balance formulation neglected effect of natural convection in the molten PCM.

Li et al. [8] studied numerically and experimentally melting process in the high-porosity copper metal foam filled with PCM. The effect of porosity and pore density on the wall temperature and the nonuniformity of temperature in the foam were investigated. Authors discussed the enhanced effective conductivity of foam matrix but also its fluid flow suppressing effect. The proposed computational model included non-equilibrium heat transfer, both viscous and inertial resistance for fluid flow and natural convection in molten PCM. Experiments outcomes revealed the temperature of the heated wall is more efficiently lowered, the porosity of the composite is lower and the pore density is lower.

The composite made of graphite matrix impregnated with PCM was used by Atkin and Farid [9] for temperature stabilization of PV cells. This solution was compared to other stabilization methods, based on passive cooling with fins. The composite material used as the heat sink, the graphite foam infused with PCM, had much higher thermal conductivity, equal to 16.6 $\mathrm{W} /(\mathrm{mK})$, than the conductivity of pure PCM, equal to $0.25 \mathrm{~W} /(\mathrm{mK})$. However, due to structure of the heat storage material, heat conduction was the only heat transfer mechanism. Experiment was conducted under artificial conditions, the insolation of maximum intensity $920 \mathrm{~W} / \mathrm{m}^{2}$, was supplied with halogen lamps. Authors observed the best performance for the system with graphite-PCM composite as the heat storage material and additional finned heat sink at the rear side. For 12 hours simulated daylight load the PV peak efficiency was higher by $2 \mathrm{pp}$ then for the reference case.

In the proposed paper four configurations of the passive temperature stabilization system are numerically investigated. Considered cases involve geometry with or without aluminium fins, with porous aluminium fins or porous aluminium layer attached to the heating surface. The numerical simulations are performed with the general purpose ANSYS Fluent software. The special treatment of mass, momentum and energy transport through porous zone, considered in two cases, is necessary so effective transport parameters in the two phase domain, like thermal conductivity and permeability are estimated. Results of numerical simulations in the simplified, two dimensional geometry, enable assessment of the heat transfer rate from the hot surface to heat container and the related temperature stabilization. Additionally, the mechanisms of heat transfer to PCM are discussed, based on the average temperature history of the hot surface.

\section{Problem statement}

In this paper the role of natural convection in molten PCM as well as enhancement of heat transfer with fins and well heat conducting porous foam are investigated.

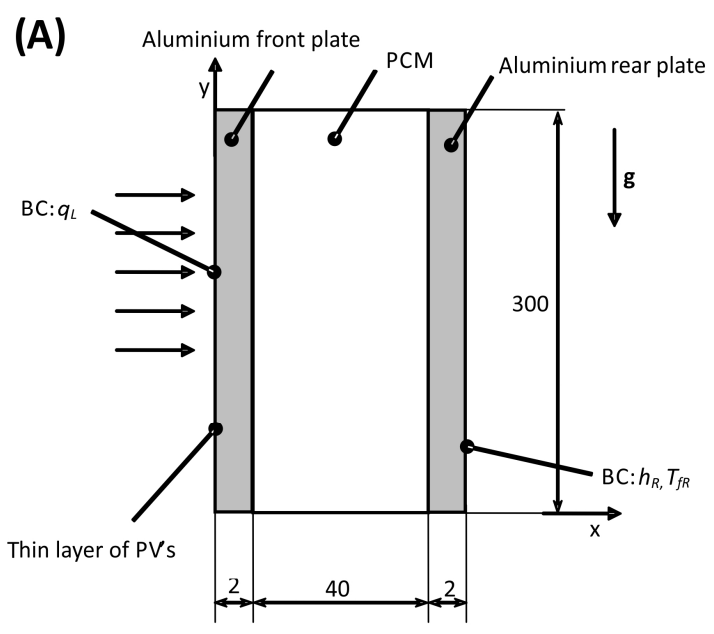

$(B, C)$

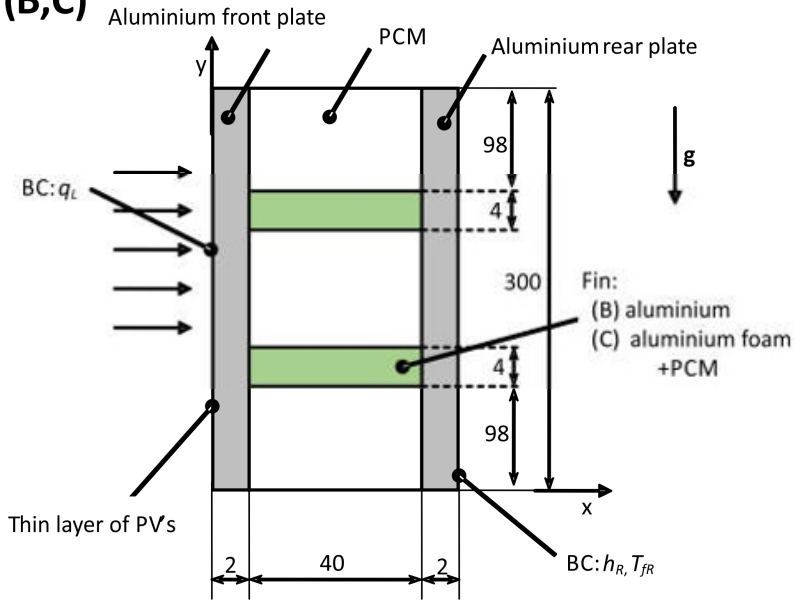

(D)

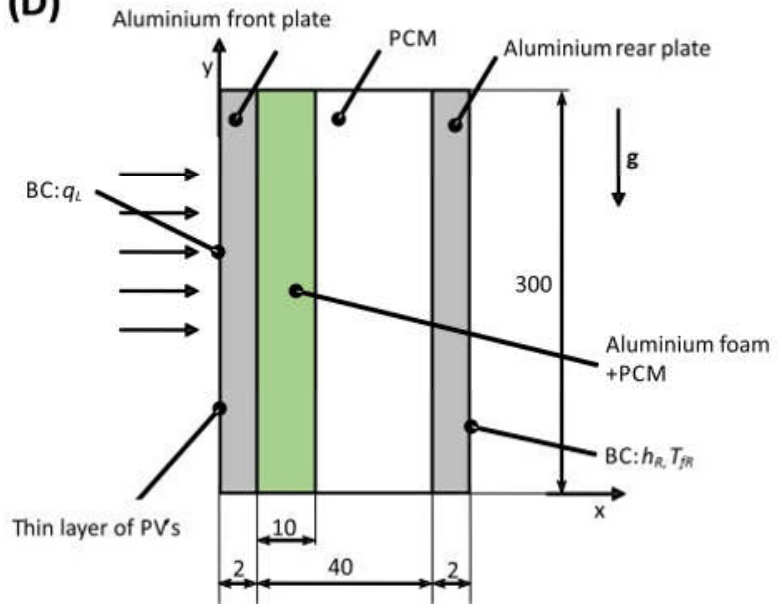

Fig. 1. Schematic of considered cases: (A) the reference geometry, (B) with two fins made of Al, (C) with two fins made of $\mathrm{Al}$ foam and (D) with $\mathrm{Al}$ foam layer attached to the hot plate.

All dimensions are given in $\mathrm{mm}$, not to scale. 
The four configurations of the passive temperature stabilization system, based on the PCM are considered (Fig. 1). Between the front and rear plates made of aluminium, the PCM is stored. The total width and height of the system are $44 \mathrm{~mm}$ and $300 \mathrm{~mm}$, respectively. In the two configurations additional fins made of aluminium (case B) or aluminium foam (case C) are present. In the case $\mathrm{D}$, the layer of aluminium foam of $10 \mathrm{~mm}$ thickness is attached to the front aluminium plate. For cases $\mathrm{C}$ and $\mathrm{D}$, the open pores and high porosity foam is used, completely filled with PCM, so both diffusive and convective heat transfer can develop in it. The perfect contact between vertical aluminium plates and fins or foam layer is assumed. At the external surface of the front plate the thin layer of PVs is fastend. The thickness of the PVs layer as well as thermal contact resistance at its back surface are neglected. At the surface of PVs, called subsequently the hot surface, the external heat flux is imposed, which reflects the insolation close to its highest value. The heat flux density, $q_{L}$, is uniform and constant in time, equal to $1000 \mathrm{~W} / \mathrm{m}^{2}$. The same value was assumed by several authors in their numerical or experimental research (e.g. [4]). In this simplified analysis the other components of heat transfer from the hod wall, namely the convective and radiative terms are neglected. Horizontal boundaries of the system are assumed to be perfectly insulated. On the external surface of the rear plate, called subsequently the cold surface, the third kind boundary condition is imposed, heat transfer coefficient, $h_{R}$, is $5 \mathrm{~W} /\left(\mathrm{m}^{2} \mathrm{~K}\right)$ and ambient air temperature, $T_{R}$, is $293.15 \mathrm{~K}$.

\section{Computational model}

The general purpose CFD software, ANSYS Fluent, has been adopted to perform numerical simulations of heat and mass transfer in the PVs temperature stabilization system. Heat transfer enhancement methods, like finning, using of well heat conducting porous foams saturated with pcm, are taken into account, so heat and mass transport phenomena through a porous zone should be taken into account.

The considered mass, momentum and energy transport equations are following:

$$
\begin{gathered}
\nabla \cdot \mathbf{V}=0 \\
\frac{\partial(\rho \mathbf{V})}{\partial t}+\nabla \cdot(\rho \mathbf{V V})=\nabla \cdot\left(\mu_{l} \nabla \mathbf{V}\right)+\mathbf{B} \\
-\varepsilon_{l} \varepsilon_{F} \nabla p-\frac{\varepsilon_{l} \varepsilon_{F} \mu_{l}}{K} \mathbf{V}-\frac{\varepsilon_{l} \varepsilon_{F} C_{I}}{K^{1 / 2}} \rho|\mathbf{V}| \mathbf{V} \\
\frac{\partial(\rho c T)}{\partial t}+\nabla \cdot(\rho c \mathbf{V T})= \\
=\nabla \cdot(k \nabla T)+\rho L \frac{\partial g_{s}}{\partial t}-\rho L \nabla \cdot\left(g_{l} \mathbf{V}_{l}\right)
\end{gathered}
$$

where the effective material properties: density, $\rho$, specific heat, $c$, viscosity, $\mu$, thermal conductivity, $k$, latent heat, $L$ are presented without indices. Momentum balance equation (2) is written in semiheuristic volumeaveraged general form [10], valid for both bulk liquid and porous zone. The one domain approach is adopted, where the highly non-uniform two-phase medium is replaced with the artificial one, which properties are determined as averaged across the phases occupying the representative elementary volume (REV). The nominal properties of PCM and aluminium are listed in the table 1. It is also assumed, the intrinsic properties of aluminium foam are the same as of the solid aluminium, however in reality those properties can slightly vary. The assumption of the equilibrium heat transfer (eq. 3) is justified with the relatively low heat fluxes and high density of pores of the foam and is sufficient for preliminary analysis.

The source term, B, in the eq. 2, express the buoyancy forces in the molten PCM. The simple Boussinesq model is taken

$$
\mathbf{B}=-\varepsilon_{l} \varepsilon_{F} \rho_{r e f} \beta \mathbf{g}\left(T-T_{r e f}\right),
$$

where the reference density, $\rho_{\text {ref, }}$ and reference temperature, $T_{r e f}$, are equal to the nominal density of the PCM and the initial temperature of the system, respectively. Product of foam porosity, $\varepsilon_{F}$, and volumetric liquid fraction of $\mathrm{PCM}, \varepsilon_{l}$, is equal to the volumetric fraction of liquid phase with respect to the total mixture volume. The same term appears in the pressure gradient, the Darcian and Forcheimer damping terms in eq. 2.

To estimate the permeability, $K$, and the intertial resistance coefficient, $C_{I}$, more detailed informations on the metal foam microstructure must be supplied. Presented model and simulations are based on the assumption, the pore number per inch (PPI) is 10. Other microstructure parameters, like pore diameter, $\mathrm{dp}$, and fiber diameted, df, are taken from Li et al. [8]. Tortuosity coefficient, $\chi$, and related characteristic length $d_{k}$, are derived with formulae [8]

$$
\begin{gathered}
\chi=2+2 \cos \left[\frac{4 \pi}{3}+\frac{\cos ^{-1}\left(2 \varepsilon_{F}-1\right)}{3}\right], \\
d_{k}=\frac{2 d_{p}}{3-\chi} .
\end{gathered}
$$

Permeability and inertial coefficient are derived with formulae [8]

$$
\begin{gathered}
K=\frac{\left(\varepsilon_{l} \varepsilon_{F}\right)^{2} d_{k}^{2}}{36(\chi-1) \chi}, \\
C_{I}=\frac{0.00212\left(1-\varepsilon_{l} \varepsilon_{F}\right)^{-0.132}}{\sqrt{K}}\left(\frac{d_{f}}{d_{p}}\right)^{-1.63},
\end{gathered}
$$

During the phase change the temperature of PCM changes in some temperature range, namely between solidus and liquidus temperatures, so the process is not isothermal as for pure substances or mixtures at the eutectic compositions. It means the zone of coexistence of solid and liquid phases appear during phase change process. Generally the properties of this mixture depend on intrinsic properties of phases and the fraction of the solid phase. It is assumed here that all material properties of solid and liquid PCM are the same and temperature independent (table 1), so all effective properties of PCM 
are constant. In two cases, $\mathrm{C}$ and $\mathrm{D}$, the permeable metal foam fully saturated with PCM occupies part of the computational domain, so effective transport properties should be determined there. Effective density, specific heat and latent heat are calculated as simple averages based on the mixture theory:

$$
\begin{gathered}
\rho=\left(1-\varepsilon_{F}\right) \rho_{F}+\varepsilon_{F} \rho_{P C M}, \\
c=\left(1-g_{F}\right) c_{F}+g_{F} c_{P C M}, \\
L=\varepsilon_{F} L_{P C M} .
\end{gathered}
$$

The effective thermal conductivity of the foam saturated with PCM was determined with formula adopted from [11]:

$$
k=0.35\left[\varepsilon_{F} k_{P C M}+\left(1-\varepsilon_{F}\right) k_{F}\right]+\frac{0.65}{\left(\frac{\varepsilon_{F}}{k_{P C M}}+\frac{1-\varepsilon_{F}}{k_{F}}\right)}
$$

Table 1. Material properties

\begin{tabular}{|l|l|l|l|}
\hline & Unit & PCM & Aluminium \\
\hline Density, $\rho$ & $\mathrm{kg} / \mathrm{m}^{3}$ & 770 & 2675 \\
\hline Specific heat, $c$ & $\mathrm{~J} /(\mathrm{kgK})$ & 2000 & 903 \\
\hline $\begin{array}{l}\text { Thermal } \\
\text { conductivity, } k\end{array}$ & $\mathrm{~W} /(\mathrm{mK})$ & 0.2 & 211 \\
\hline Viscosity, $\mu$ & $\mathrm{kg} /(\mathrm{ms})$ & 0.02 & n.a. \\
\hline $\begin{array}{l}\text { Volumetric } \\
\text { expansion } \\
\text { coefficient, } \beta_{T}\end{array}$ & $1 / \mathrm{K}$ & 0.001 & n.a. \\
\hline $\begin{array}{l}\text { Melting } \\
\text { temperature, } T_{M}\end{array}$ & ${ }^{\circ} \mathrm{C}$ & $27-29$ & n.a. \\
\hline Latent heat, $L$ & $\mathrm{~J} / \mathrm{kg}$ & 220000 & n.a. \\
\hline
\end{tabular}

The considered transport equations (eq. 1-3) supplied with the closure relations (eq. 4-12) were solved with the ANSYS Fluent software. The non-stationary equations were integrated with the fully implicit scheme with time step $0.01 \mathrm{~s}$. The second order upwind scheme was used for all equations. The pressure and velocity fields were coupled with the PISO scheme.

\section{Verification}

The implementation of proposed model in the commercial software Fluent was werified with numerical results presented by Beckermann and Viskanta [12]. The considered case is the melting of gallium in the porous matrix of glass beads in a square cavity heated from the left wall. All material properties and boundary conditions were adopted from the referred paper [12]. Both viscous resistance and inertial resistance terms were taken into account according to formulations presented in [12]. Domain was discretized with the uniform mapped grid of 10000 control volumes. The fully implicit non-iterative time advancing PISO scheme was used with uniform time sted $0.01 \mathrm{~s}$. The second order upwind scheme was used for each balance equation.
Gradients were determined with the Least Squares Cell Based scheme. The comparison of phase interface predited for several time instants with ANSYS Fluent (solid lines) and numerical (dashed lines) taken from the paper are presented in Fig. 2. Results reveal very good conformity.

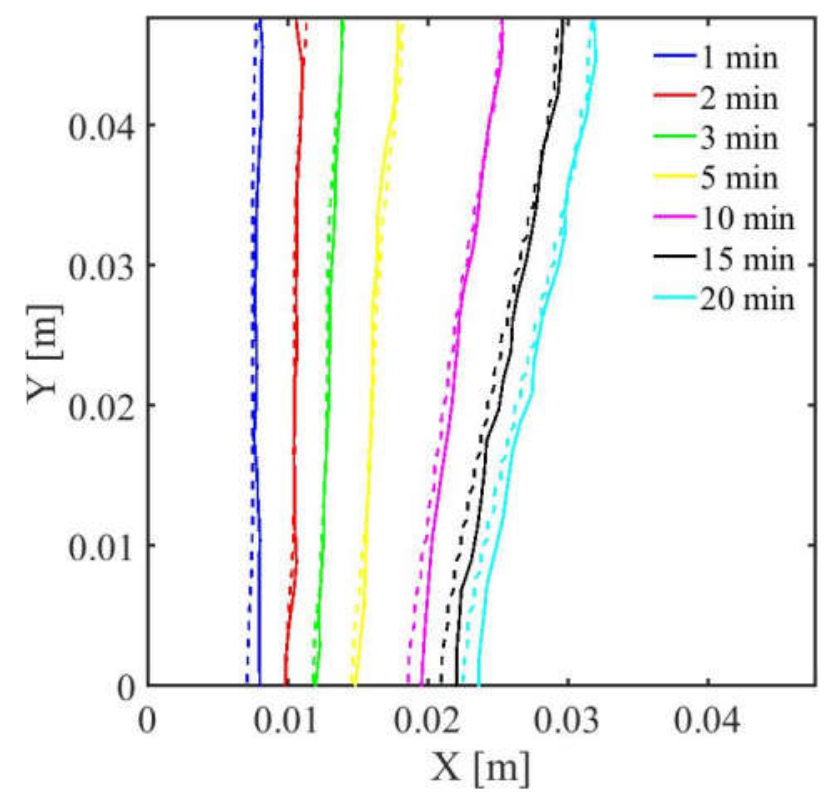

Fig. 2. Comparison of interface locations predicted numerically, present work - solid lines and the reference data [12] - dashed line.

\section{Results and discussion}

The presented model (eq. 1-3) supplied with closure relations (eq. 4-8) and definitions of effective metal properties (eq. 9-12) was used to simulate the response of systems A-D to thermal loading as were detailed in the previous chapters.

The comparison of temperature distributions (Fig. 3) after $1 \mathrm{~h}$ of heating reveals that fins made of aluminium (case B) or aluminium foam (case C) considerably promote heat transfer to the back side of the container. So the maximum temperature of the front plate is visibly reduced to $309.1 \mathrm{~K}$ for the case $\mathrm{B}$ and $312.78 \mathrm{~K}$ for the case Ccompared to $318.66 \mathrm{~K}$ for the case A. Presence of the aluminium foam slab attached to the front plate (case D) also reduces the maximum temperature but it is slightly higher than in cases B and C. Presence of fins (cases B and C) considerably equalize temperature distribution in the PCM, heat is more efficiently dissipated in the bottom part of the container. Similar effect of temperature equilization is observed in the case D. Temperature across the metal foam layer is nearly constant, but it increases towards its upper part. Beyond the foam layer the single vortex is formed, similar to those present in the case $\mathrm{A}$, but rate of melting in the upper part (case D) is lower than observed in the case A (Fig. 4). Contrary, the predicted melting rate in the bottom part is the highest for case D (Fig. 4).

This tendency for temperature equalization is confirmed in plots of temperature variation in time (Fig. 5), 
determined at three points, $\mathrm{P}_{1}, \mathrm{P}_{2}, \mathrm{P}_{3}$, located in the halt height of the domain (Table 2).
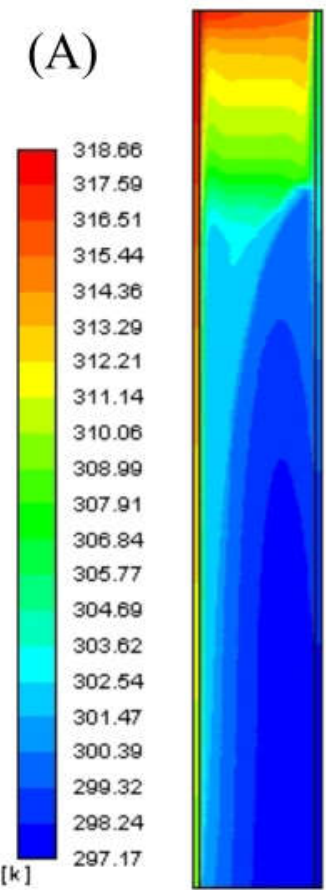

(B)
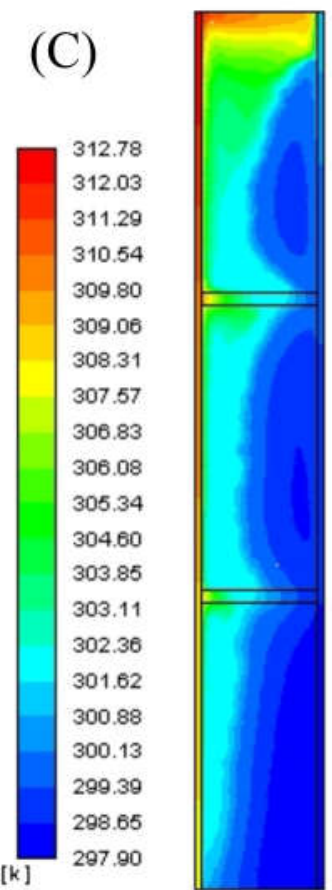

(D)

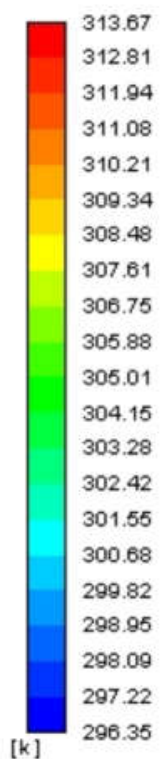

Fig. 3. Comparison of temperature distributions in the PCM container after $1 \mathrm{~h}$ of heating.

The highest surface temperature is obtained for the case A (blue solid line). The existence of the evident maximum in temperature, predicted at point $\mathrm{P}_{1}$, is observed for cases $\mathrm{A}$ and $\mathrm{D}$, where single vortex forms in the molten PCM. It is related with the conductive only heat transfer mode present in the starting period of heating, where the width of the molted PCM layer is too low for development of natural convection. It is particularly visible in the central and bottom part of the container. The time period, when the heat transfer is convective only, is reduced considerably in cases B and $\mathrm{C}$ due to more efficient heat transfer to the rear part of the container and additional PCM melting close ti fins and rear plate. After the convective flow develops, the temperature in $\mathrm{P}_{1}$ is visibly reduced. Additionally, for the case $\mathrm{D}$, the temperature in $\mathrm{P}_{1}$ is initially reduced due to effective heat transport through the composite layer.
(A)

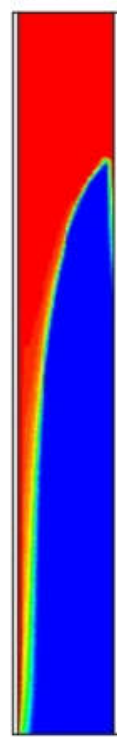

(C)

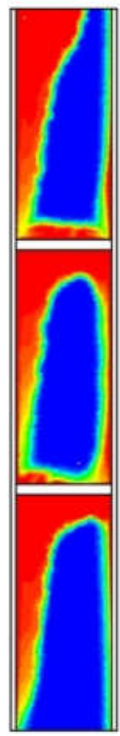

(D)

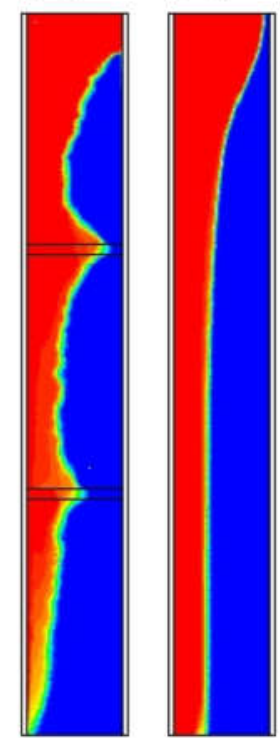

Fig. 4. Comparison of liquid fraction distributions in the PCM container after $1 \mathrm{~h}$ of heating.

Table 2. Coordinates of probe points. The origin of the global Cartesian coordinate system is in the left, bottom corner of the

\begin{tabular}{|l|l|l|}
\multicolumn{3}{l}{ domain. } \\
\hline & $X[\mathrm{~mm}]$ & $\mathrm{Y}[\mathrm{mm}]$ \\
\hline $\mathrm{P}_{1}$ & 4 & 150 \\
\hline $\mathrm{P}_{2}$ & 10 & 150 \\
\hline $\mathrm{P}_{3}$ & 22 & 150 \\
\hline
\end{tabular}

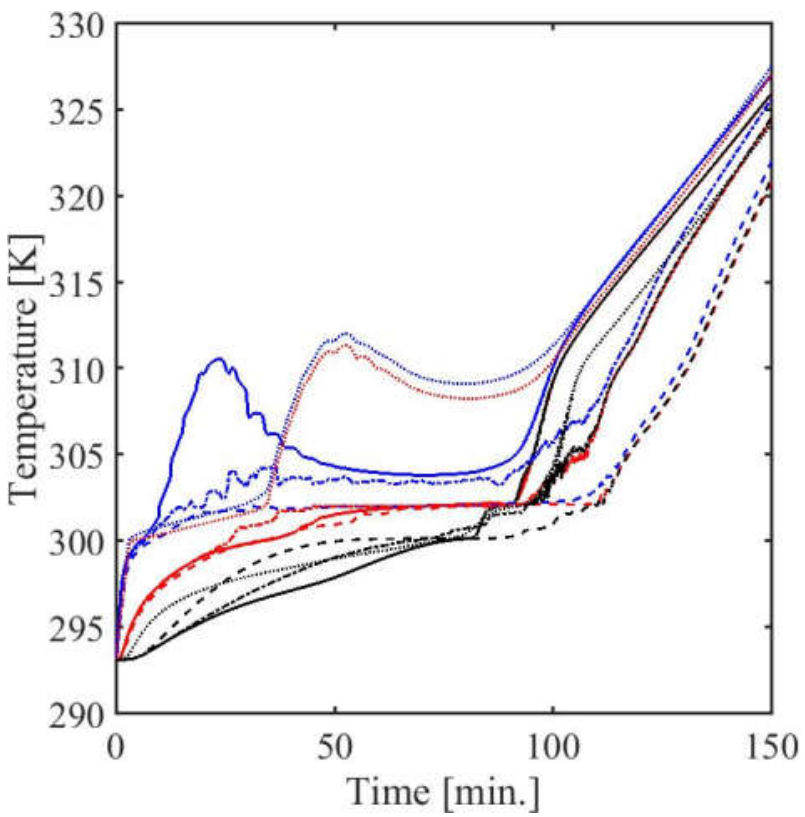

Fig. 5. Time evolution of temperature measured in three probe points: $\mathrm{P}_{1}$ - blue line, $\mathrm{P}_{2}-$ red line and $\mathrm{P}_{3}-$ black line. For case $\mathrm{A}$ - solid line, case B - dashed line, case $\mathrm{C}$ - dash-dot line and case $\mathrm{D}$ - dotted line. 
Variations in time of the average temperatures calculated on the heating wall are presented in Fig. 6. The shapes of curves are similar to those predicted in $\mathrm{P}_{1}$ (Fig. 5). The local maximum in temperature is evident for cases $\mathrm{A}$ and $\mathrm{D}$, but the latter is much more lower so the temperature stabilization is better, especially in the first period of heating. The best temperature stabilization configuration is the $\mathrm{B}$ case.

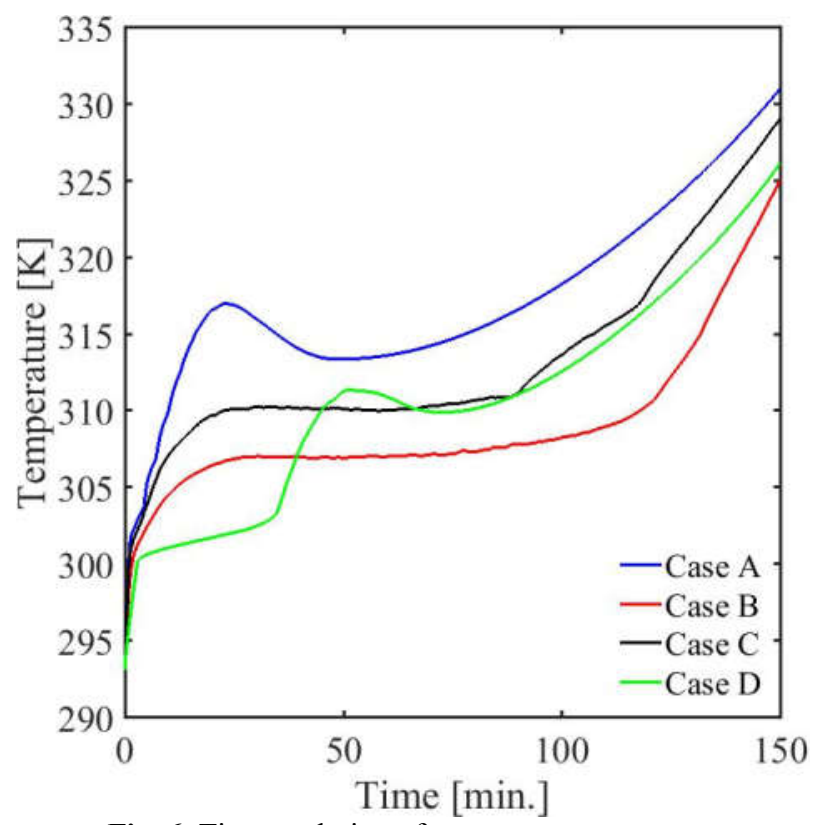

Fig. 6. Time evolution of an average temperature of the heating surface.

\section{Conclusions}

In the proposed paper the performance of four configurations of the heat sink used for temperature stabilization are discussed. The dominant heat transfer mode can be easily deduced based on the average temperature predicted on the heating wall. The retarded heat transport to PCM due to long time period necessary to develop natural convection in the molten PCM manifests with maximum in the temperature plot. It can be avoided as fins are present (cases B and C), the rate of melting is higher due to simultaneous melting close to the fins and the rear wall. It is worth noting, the fins connecting the front and rear walls are highly effective, melting occurs also close to the real wall (Fig. 5). Taking into account the average temperature on the heated wall the best considered arrangement, assuring the lowest average front temperature, is the $\mathrm{B}$ case. The issue of validity of the equilibrium model for heat transport will be addressed in the future research. Based on the werification of the model with respect to positively validated numerical data presented in [12], the utilization of the equilibrium model is justified.

\section{References}

1. E. Radziemska, Renew. Energ. 28, 1 (2003)

2. A. Hasan, S.J. McCormack, M.J. Huang, B. Norton, Sol. Energy 84, 1601 (2010)
3. T.M. Sathe, A.S. Dhoble, Renew. Sust. Energ. Rev. 76, 645 (2017)

4. M.J. Huang, P.C. Eames, B. Norton, Int. J. Heat Mass Tran. 47, 2715 (2004)

5. M.J. Huang, P.C. Eames, B. Norton, Sol. Energy 80, 1121 (2006)

6. M.J. Huang, P.C. Eames, B. Norton, N.J. Hewitt, Sol. Energ. Mat. Sol. C. 95, 1598 (2011)

7. C.Y. Zhao, W. Lu, Y. Tian, Sol. Energy 84, 1402 (2010)

8. W.Q. Li, Z.G. Qu, Y.L. He, W.W. Tao, Appl. Therm. Eng. 37, 1 (2012)

9. P. Atkin M.M. Farid, Sol. Energy 114, 217 (2015)

10. M. Kaviany, Principles of heat transfer in porous media, 2nd edition, Springer, New York (1995).

11. A. Bhattacharya, V.V. Calmidi, R.L. Mahajan, Int. J. Heat Mass Tran. 45, 1017 (2002)

12. C. Beckermann, R. Viskanta, Int. J. Heat Mass transfer, 31, 35 (1988) 